\title{
Tumor necrosis factor- $\alpha$ impairs oligodendroglial differentiation through a mitochondria-dependent process
}

\author{
M Bonora ${ }^{1}$, E De Marchi ${ }^{1,7}$, S Patergnani ${ }^{1,7}$, JM Suski ${ }^{1,2,7}$, F Celsi $^{3}$, A Bononi ${ }^{1}$, C Giorgi ${ }^{1}$, S Marchi ${ }^{1}$, A Rimessi ${ }^{1}$, J Duszyński ${ }^{2}$, \\ T Pozzan ${ }^{4,5,6}$, MR Wieckowski ${ }^{2,8}$ and P Pinton ${ }^{\star, 1,8}$
}

\begin{abstract}
Mitochondrial defects, affecting parameters such as mitochondrial number and shape, levels of respiratory chain complex components and markers of oxidative stress, have been associated with the appearance and progression of multiple sclerosis. Nevertheless, mitochondrial physiology has never been monitored during oligodendrocyte progenitor cell (OPC) differentiation, especially in OPCs challenged with proinflammatory cytokines. Here, we show that tumor necrosis factor alpha (TNF- $\alpha$ ) inhibits OPC differentiation, accompanied by altered mitochondrial calcium uptake, mitochondrial membrane potential, and respiratory complex I activity as well as increased reactive oxygen species production. Treatment with a mitochondrial uncoupler (FCCP) to mimic mitochondrial impairment also causes cells to accumulate at the progenitor stage. Interestingly, AMP-activated protein kinase (AMPK) levels increase during TNF- $\alpha$ exposure and inhibit OPC differentiation. Overall, our data indicate that TNF- $\alpha$ induces metabolic changes, driven by mitochondrial impairment and AMPK activation, leading to the inhibition of OPC differentiation.
\end{abstract}

Cell Death and Differentiation (2014) 21, 1198-1208; doi:10.1038/cdd.2014.35; published online 21 March 2014

Multiple sclerosis (MS) is a neurological disorder of the central nervous system that is characterized by demyelination and neurodegeneration. Although the pathogenesis of MS is not completely understood, various findings suggest that immune-mediated loss of myelin and different types of mitochondrial dysfunction are associated with this disease. ${ }^{1}$ Mitochondria are often described as cellular powerhouses that utilize oxygen to produce adenosine triphosphate (ATP), a molecule that is critical for most cellular functions. ${ }^{2}$ In addition, mitochondria are the major sites of the intracellular production of highly reactive free radicals that, if not neutralized, alter cellular metabolism and damage cellular components. ${ }^{3}$

In several studies, mitochondrial dysfunction has been reported to be frequently associated with demyelination, whereas proper function is required for correct oligodendrocyte differentiation and myelination. ${ }^{4,5}$ Furthermore, there is in vitro evidence that cytokine-induced oligodendrocyte injury involves mitochondrial dysfunction. ${ }^{6}$ One cytokine that is of particular interest in MS is tumor necrosis factor alpha
$(T N F-\alpha)$. Evidence implicating TNF- $\alpha$ in the underlying pathology of MS includes: (i) reports that MS patients have elevated TNF- $\alpha$ levels at the sites of active MS lesions at autopsy, ${ }^{7}$ (ii) reports that TNF- $\alpha$ levels are elevated in the cerebrospinal fluid and serum of individuals with MS compared with unaffected individuals and that these TNF- $\alpha$ levels correlate with the severity of the lesions. ${ }^{8,9}$

Moreover, it has been widely reported that TNF- $\alpha$ is able to impair oligodendrocyte differentiation and that in leukemia cell lines, TNF- $\alpha$-induced cell death requires impairments in the activity of mitochondrial respiratory chain complex I. Complex $\mathrm{I}$ is strategically important for regulating ATP synthesis and is one of the most important sources of reactive oxygen species (ROS) within cells. ${ }^{10}$ Despite this evidence, the relationships between mitochondrial physiology, TNF- $\alpha$, and oligodendrocyte differentiation have not yet been examined. This study addressed the hypothesis that the impairment of oligodendrocyte differentiation caused by TNF- $\alpha$ exposure is causally linked to altered mitochondrial physiology.

${ }^{1}$ Department of Morphology, Surgery and Experimental Medicine, Section of Pathology, Oncology and Experimental Biology, Interdisciplinary Center for the Study of Inflammation (ICSI), Laboratory for Technologies of Advanced Therapies (LTTA), University of Ferrara, Ferrara, Italy; ${ }^{2}$ Department of Biochemistry, Nencki Institute of Experimental Biology, Warsaw, Poland; ${ }^{3}$ Institute for Maternal and Child Health, IRCCS Burlo Garofolo, Trieste, Italy; ${ }^{4}$ Istituto Veneto di Medicina Molecolare, Fondazione per la Ricerca Biomedica Avanzata, Padua, Italy; ${ }^{5}$ Dipartimento di Scienze Biomediche, Università di Padova, Padua, Italy and ${ }^{6}$ Consiglio Nazionale delle Ricerche, Istituto di Neuroscienze, Sezione di Padova, Padua, Italy

*Corresponding author: P Pinton, Signal Transduction Lab, c/o CUBO, via Fossato di Mortara 70, I-44121 Ferrara, Italy. Tel: + 39532455802 ; Fax: + 39532455351 ; E-mail: pnp@unife.it

${ }^{7}$ These authors contributed equally to this work.

${ }^{8}$ These authors share senior co-authorship.

Abbreviations: AMPK, AMP-activated protein kinase; ATP, adenosine triphosphate; bFGF, basic fibroblast growth factor; $\mathrm{Ca}^{2+}$, calcium; CCh, carbachol; cytAEQ, cytoplasmic aequorin; FCCP, carbonyl cyanide 4-(trifluoromethoxy)phenylhydrazone; mTOR, mechanistic target of rapamycin; FRET, Förster resonance energy transfer; GFAP, glial fibrillary acid protein; IFN- $\gamma$, interferon gamma; MBP, myelin basic protein; mtAEQ, mitochondrial aequorin; MS, multiple sclerosis; NG2, chondroitin sulfate proteoglycan4; OPCs, oligodendrocyte progenitor cells; OSP, oligodendrocyte-specific protein; O4, oligodendrocyte marker 4; PDGF, platelet-derived growth factor; ROS, reactive oxygen species; TMRM, tetramethylrhodamine methyl ester; TNF- $\alpha$, tumor necrosis factor alpha; T3, triiodothyronine

Received 20.7.13; revised 22.1.14; accepted 06.2.14; Edited by L Scorrano; published online 21.3.14 


\section{Results}

The ability of TNF- $\alpha$ to impair oligodendrocyte progenitor cell (OPC) differentiation in an in vitro system was examined following the protocol described by Chen et al. ${ }^{11}$ Immunostaining was performed using markers of the total oligodendrocyte population (oligodendrocyte-specific protein, or OSP) and for astrocytes (glial fibrillary acid protein, or GFAP) to verify the purity of the culture, which was found to contain $95.01 \pm 1.0067 \% \mathrm{OSP}^{+}$cells and negligible numbers of $\mathrm{GFAP}^{+}$cells. After 7 days of culture, the OPCs were treated for $24 \mathrm{~h}$ with a sublethal concentration of TNF- $\alpha$, or $10 \mathrm{ng} / \mathrm{ml}$, as suggested in a previous study. ${ }^{12}$ Subsequently, the growth factors present within the medium, platelet-derived growth factor (PDGF), and basic fibroblast growth factor (bFGF) (which are fundamental to OPC proliferation), were replaced by the thyroid hormone triiodothyronine (T3) to stimulate cell differentiation. After 5 days, the cells were fixed and immunostained for markers of different stages of oligodendrocyte maturation. On the basis of the literature, three different antigens were selected in the three stages of differentiation: the chondroitin sulfate proteoglycan (NG2) was chosen to mark the precursor stage, oligodendrocyte marker 4 (O4) was chosen to mark immature oligodendrocytes, and myelin basic protein (MBP) was chosen to mark fully differentiated cells. ${ }^{13}$ All of the cells were co-stained for $\mathrm{OSP}$, thus restricting the analysis to oligodendrocyte populations only and avoiding artifacts from any possible contaminants. The cells were then visualized using an image-based automated system and classified by their antigen positivity status. A mixed population of cells at different stages was observed, with proportions varying depending on the analyzed culture. The average values were $13 \%$ for progenitors (S.E.M. 1.87), 25\% for immature oligodendrocytes (S.E.M. 4.997), and $19 \%$ for adult oligodendrocytes (S.E.M. 4.17). Owing to the choice of markers of the different stages, the sum of the values was not expected to reach $100 \%$ (Figure 1ai).

In the presence of TNF- $\alpha$ at $10 \mathrm{ng} / \mathrm{ml}$, several variations in the proportions of cells at different stages were observed. In particular, a reproducible reduction in the proportions of $\mathrm{MBP}^{+} / \mathrm{OSP}^{+}$cells and $\mathrm{O}^{+} / \mathrm{OSP}^{+}$cells was observed (36.78\%, S.E.M. 8.63 and $30.33 \%$, S.E.M. 19.06, respectively), with an accompanying enrichment of $\mathrm{NG}^{+} / \mathrm{OSP}^{+}$ cell levels (41.71\%, S.E.M. 14.67), suggesting that early inhibition of the differentiation process was occurring (Figure 1a(i) and (ii)). The inhibition of OPC differentiation was also confirmed by monitoring the level of the adult oligodendrocyte marker MBP (Figure 1b).

A terminal deoxynucleotidyl transferase dUTP nick end labeling (TUNEL) assay was used to test the ability of TNF- $\alpha$ to induce cell death. We observed that at a dose of $10 \mathrm{ng} / \mathrm{ml}$, TNF- $\alpha$ did not induce a significant increase in the number of TUNEL-positive nuclei in the $\mathrm{OSP}^{+}$cells. In contrast, a progressive increase in the TNF- $\alpha$ concentration (to 50 or $100 \mathrm{ng} / \mathrm{ml}$ ) caused a linear increase in the number of apoptotic cells (Figure 1c), indicating that the variation in the proportions of differentiation stages was not due to the induction of apoptosis.

The final events in oligodendrocyte differentiation are the production of myelin and the ensheathment of axons.
Therefore, we examined whether TNF- $\alpha$ might alter the activity of differentiating cells in addition to blocking differentiation initiation and reducing the number of differentiating OPCs. For this study, we created a mixed glial culture enriched in astrocytes, mature oligodendrocytes, OPCs, and neurons, as indicated by western blotting for the specific markers NG2 (for oligodendrocyte progenitors), $\beta$-tubulin III (for neurons), and MBP (for mature oligodendrocytes) (Figure 2a). The mixed glial cultures were pretreated with TNF- $\alpha$ at $10 \mathrm{ng} / \mathrm{ml}$ and then exposed to the thyroid hormone T3 to promote oligodendrocyte differentiation, which was monitored by western blotting (Figure 2a). We observed an increase in NG2 levels, with a concomitant reduction in MBP expression, compared with expression in control cultures treated with T3 alone. Furthermore, to ensure that there was no misinterpretation of the data due to increased cell death, we measured the levels of the apoptotic marker cleaved PARP (cPARP) and found no significant variation in this parameter. The number of differentiating OPCs was also monitored by immunostaining, as previously described, involving counting the proportion of $\mathrm{OSP}^{+}$cells that were also $\mathrm{MBP}^{+}$. Again, a reduced number of $\mathrm{MBP}^{+} / \mathrm{OSP}^{+}$cells was detected after exposure to TNF- $\alpha$ (Figure $2 b$ ).

Next, we used 3D confocal imaging to measure the extent of axonal myelination in a mixed glial and OPC co-culture. ${ }^{14}$ OPCs and mixed glia were grown separately and treated with $10 \mathrm{ng} / \mathrm{ml} \mathrm{TNF-} \alpha$ or vehicle. After $24 \mathrm{~h}$, the OPCs were detached and seeded into the mixed glial culture, then, by addition of the thyroid hormone T3 we induced oligodendrocyte differentiation. The cultures were then immunostained for the axonal marker $\beta$-tubulin III and with the myelin marker MBP. The portions of the images containing axons in which the markers were either isolated or colocalized were measured as the index of myelination. ${ }^{14} \mathrm{~A}$ large number of axons was detected in the control cultures (Figure 2c), in which nearly $25 \%$ of the $\beta$-tubulin III signals colocalized with MBP. In contrast, in cultures exposed to TNF- $\alpha$, the proportion of colocalization decreased to only $12 \%$.

To determine the mechanism underlying the selective impairment of OPC differentiation, we examined mitochondrial physiology during OPC exposure to $10 \mathrm{ng} / \mathrm{ml} \mathrm{TNF-} \alpha$ for $24 \mathrm{~h}$ before differentiation induction. Our group and others have successfully used mitochondrial calcium $\left(\mathrm{Ca}^{2+}\right)$ measurements utilizing recombinant aequorin as a tool to investigate mitochondrial physiology. ${ }^{15,16}$

OPCs were then infected with an adenoviral vector carrying cDNA for a mitochondria-targeted mutant aequorin to investigate the effect of TNF- $\alpha$ on mitochondrial $\mathrm{Ca}^{2+}$ homeostasis.

The resting $\mathrm{Ca}^{2+}$ level within the mitochondrial matrix $\left(\left[\mathrm{Ca}^{2+}\right]_{\mathrm{m}}\right)$ was not significantly affected; however, the increased amplitude of $\left[\mathrm{Ca}^{2+}\right]_{\mathrm{m}}$ elicited by the muscarinic agonist carbachol (CCh) (Figure $3 a$ ) was drastically reduced in cells exposed to TNF- $\alpha$. To verify whether this effect of TNF- $\alpha$ was dependent on a defect in mitochondrial $\mathrm{Ca}^{2+}$ handling or was due to a reduction in the amplitude of $\mathrm{Ca}^{2+}$ mobilization caused by $\mathrm{CCh}$, in parallel experiments, the cells were infected with viruses encoding cytoplasmic aequorin (cytAEQ). As shown in Figure 3b, CCh addition rapidly and transiently increased the cytosolic $\mathrm{Ca}^{2+}$ concentration 
a

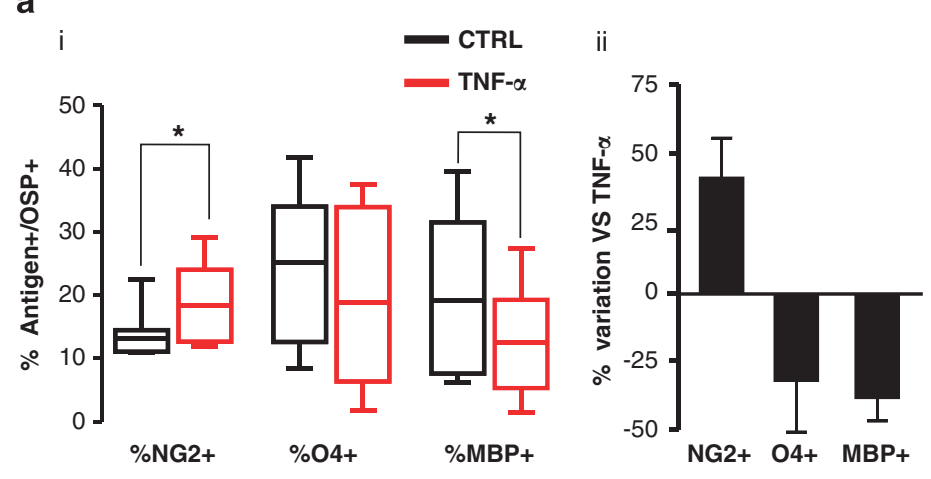

b

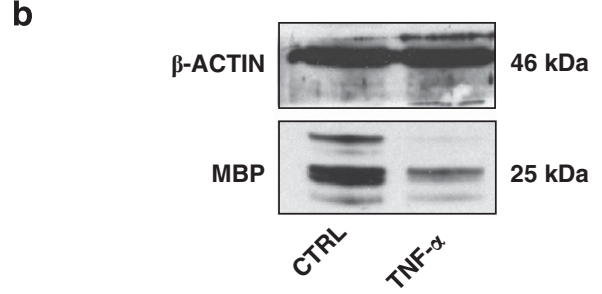

C

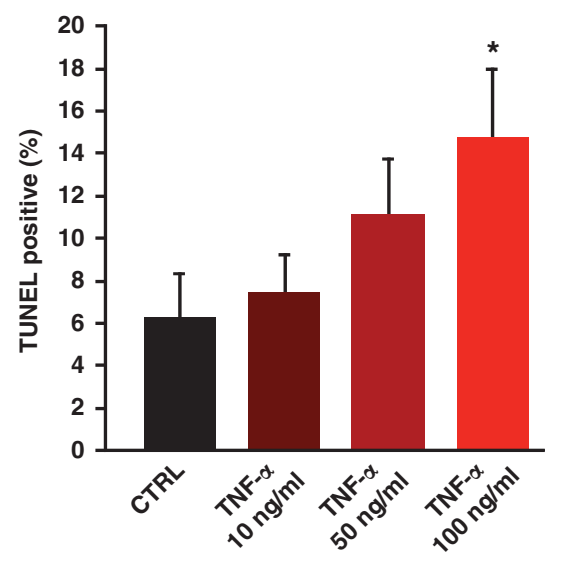

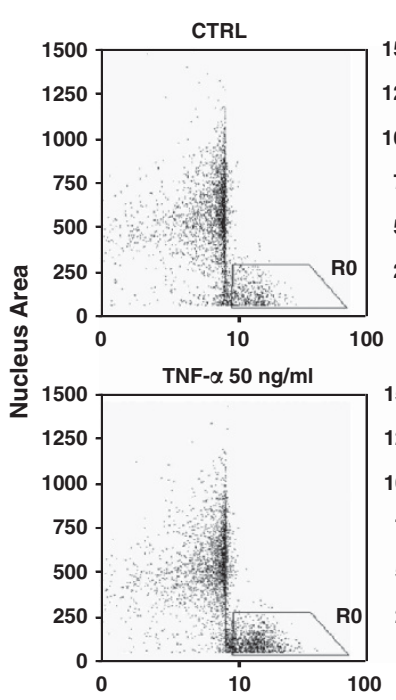
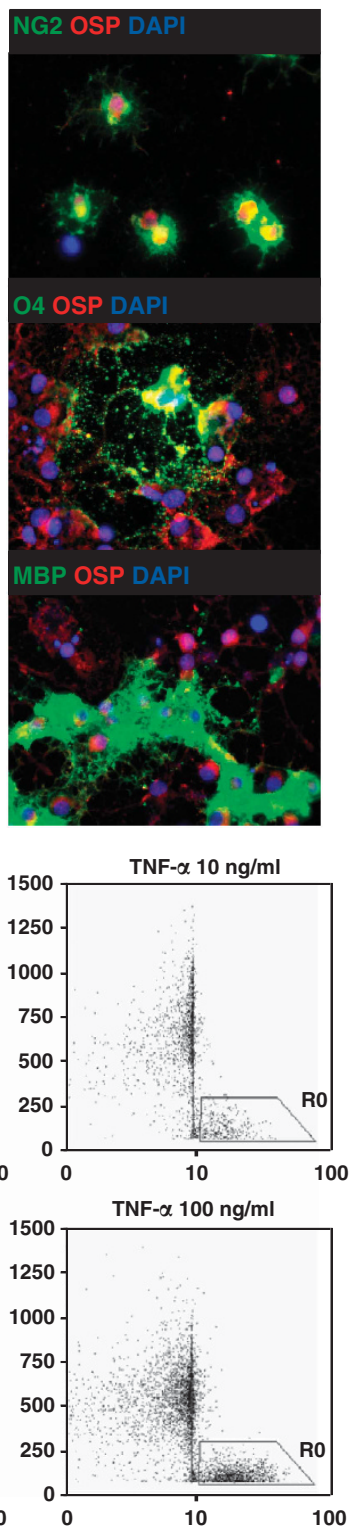

Log TUNEL intensity

Figure 1 (a(i)) Differentiation assay in control (CTRL) and TNF- $\alpha$-pretreated OPCs induced to differentiate by 5 days of exposure to the thyroid hormone T3. The proportions of different markers were determined on oligodendrocytes expressing the marker OSP. Percentages (mean \pm S.E.M.) of NG2 ${ }^{+}$cells (progenitors): CTRL, $13.2 \pm 1.87 \%$; TNF- $\alpha, 18.43 \pm 2.64 \%$; O4 ${ }^{+}$cells (intermediate stage): CTRL, $25.14 \pm 4.99 \% ;$ TNF- $\alpha, 18.85 \pm 6.04 \%$; and MBP ${ }^{+}$cells (adult): CTRL, $19.21 \pm 4.73 \%$; TNF- $\alpha$ $12.51 \pm 3.39 \%$. Representative images of cells expressing different markers are shown in the right panel. * Significant at $P<0.05$; line, mean; bars, maximum and minimum values. The boxes envelop the 25th to the 75th percentile of the assayed population, with $n=6$ independent cultures. (a(ii)) Percentage variation in proportions (mean \pm S.E.M.) within assayed cultures between CTRL and TNF- $\alpha$-treated cells: NG2 ${ }^{+}: 41.71 \pm 14.67 \%$; $04+$ : $-30.61 \pm 19.06 \%$; and MBP ${ }^{+}:-36.78 \pm 8.63 \%$. Representative images of cells expressing different markers are shown in the lower panel. (b) Expression levels of the adult oligodendrocyte marker MBP in OPCs after 5 days of differentiation induction. (c) Proportions (mean \pm S.E.M.) of TUNEL-positive OPCs after 5 days of differentiation in the CTRL $(6.23 \pm 3.697 \%)$, $10 \mathrm{ng} / \mathrm{ml}$ TNF- $\alpha$ $(7.43 \pm 1.85 \%), 50 \mathrm{ng} / \mathrm{ml} \mathrm{TNF}-\alpha(11.143 \pm 3.89 \%)$, and $100 \mathrm{ng} / \mathrm{ml} \mathrm{TNF}-\alpha(14.76 \pm 3.24 \%)$ groups. ${ }^{*}$ Significant at $P<0.05$; bars $=$ S.E.M. from three independent cultures

$\left(\left[\mathrm{Ca}^{2+}\right]_{\mathrm{c}}\right)$ both in control cells and in OPCs pretreated with TNF- $\alpha 10 \mathrm{ng} / \mathrm{ml}$ for $24 \mathrm{~h}$ ). No significant differences were observed between control and TNF- $\alpha$-treated cells, suggesting that intracellular $\mathrm{Ca}^{2+}$ stores were not affected by TNF- $\alpha$. We also verified whether TNF- $\alpha$ could acutely alter cellular $\mathrm{Ca}^{2+}$ homeostasis. To this end, OPCs were loaded with the $\mathrm{Ca}^{2+}$-sensitive dye Fura-2 and monitored by fluorescence microscopy during acute TNF- $\alpha$ exposure. For more than
10 min following TNF- $\alpha$ administration, no significant variation in the Fura-2 signal was observed (Supplementary Figure S1).

To verify that the variations in $\left[\mathrm{Ca}^{2+}\right]_{\mathrm{m}}$ were not due to alterations in cells contaminating the OPC population (i.e., astrocytes), $\mathrm{Ca}^{2+}$ waves were monitored in single cells using the $\mathrm{Ca}^{2+}$-sensitive, Förster resonance energy transfer (FRET)-based probe mtD3cpv. ${ }^{17}$ OPCs were easily distinguished from other cells based on their morphology. 
a

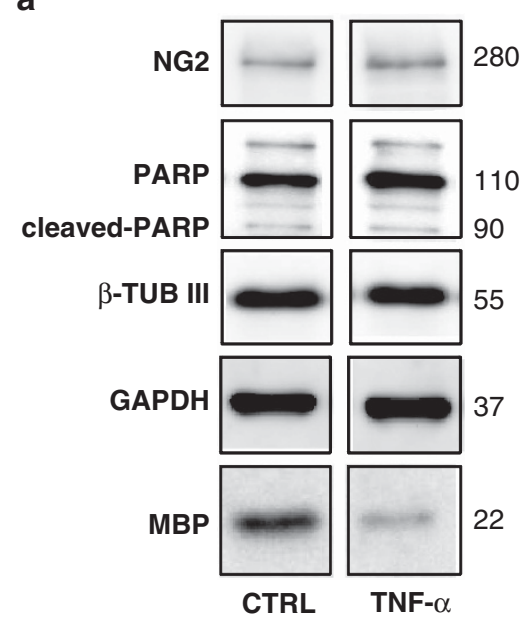

b
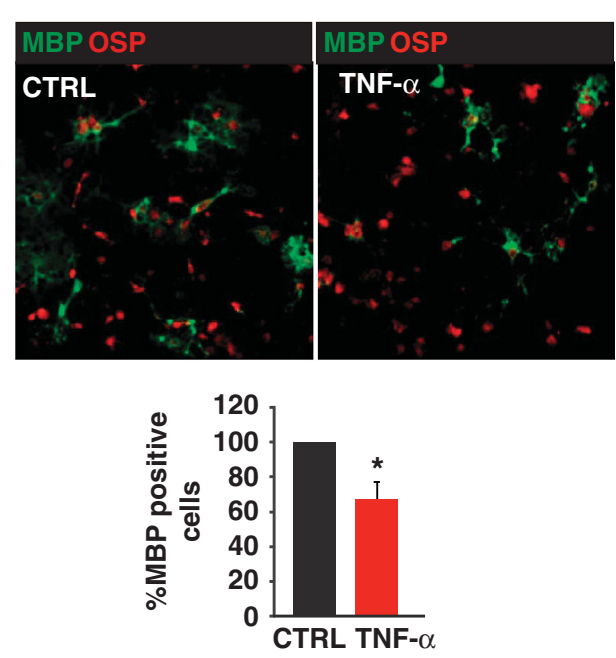

c
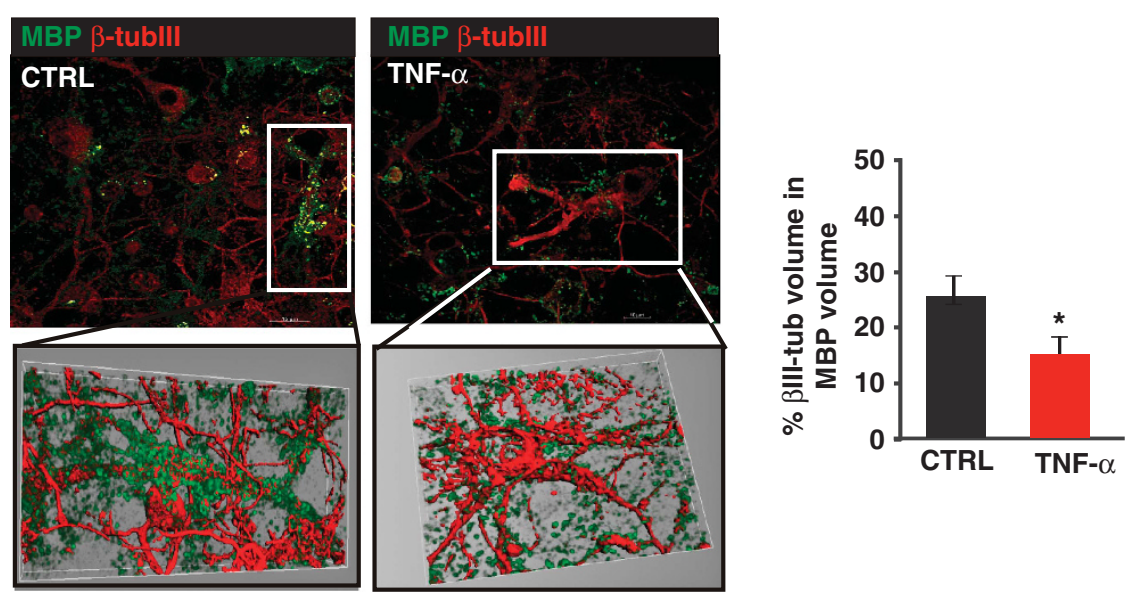

Figure 2 Expression levels of markers of cell types within the mixed glial population (NG2 for OPCs, $\beta$-tubulin IIII for neurons, and MBP for adult oligodendrocytes) and of the apoptotic marker PARP (whole and cleaved forms) (a) in control (CTRL) and TNF- $\alpha$-exposed cells (b). Immunostaining for MBP and OSP in a mixed glial culture and relative quantification of the proportion of $\mathrm{MBP}^{+}$cells among all $\mathrm{OSP}^{+}$cells. The values are expressed as the percentage variation compared with the CTRL (mean \pm S.E.M.: TNF- $\alpha, 64.2 \pm 4.98 \%$ ). (c) Maximum intensity projection (top) and 3D volume rendering (bottom) of co-cultured glia and OPCs stained for MBP (myelin marker) and $\beta$-tubulin III (axonal marker) during induction of the differentiation of CTRL and TNF- $\alpha$-exposed cells by the thyroid hormone T3. The myelination activity was quantified as the proportion of $\beta$-tubulin III signal colocalizing with MBP signal (mean \pm S.E.M.: CTRL, $26.6 \pm 3.15 \%$; TNF- $\alpha, 14.9 \pm 2.82 \%$ ). ${ }^{*}$ Significant at $P<0.05$; bars, S.E.M. from four independent cultures

This approach confirmed that the capacity of mitochondria to take up $\mathrm{Ca}^{2+}$ in response to CCh was impaired by exposure to TNF- $\alpha$. However, single-cell measurements that allowed precise measurements of $\mathrm{Ca}^{2+}$ uptake and release kinetics showed that TNF- $\alpha$ treatment reduced the amplitude, but not the speed, of the changes in $\mathrm{Ca}^{2+}$ levels (Supplementary Figure S2). We next investigated the effects of interferon gamma (IFN- $\gamma$ ) on mitochondrial $\mathrm{Ca}^{2+}$ homeostasis. It is well known that this cytokine, similar to TNF- $\alpha$, has a key role in MS and other demyelinating disorders. ${ }^{18}$ OPCs were treated with $20 \mathrm{ng} / \mathrm{ml} \mathrm{IFN}-\gamma$ for $24 \mathrm{~h}$, and the $\left[\mathrm{Ca}^{2+}\right]_{\mathrm{m}}$ changes elicited by $\mathrm{CCh}$ stimulation were investigated. However, no significant difference in $\left[\mathrm{Ca}^{2+}\right]_{\mathrm{m}}$ was observed between IFN- $\gamma$-treated and control cells (Supplementary Figure S3a). Next, we investigated whether TNF- $\alpha$ affects mitochondrial $\mathrm{Ca}^{2+}$ homeostasis in other glial cell types, such as astrocytes. To address this issue, a mixed glial culture was transfected with mitochondrial aequorin (mtAEQ) using a liposome approach. Owing to the elevated abundance of astrocytes in this mixed glial culture (and the elevated toxicity mediated by liposomes in OPCs), most of the cells carrying aequorin were astrocytes (verified in parallel by staining with anti-GFAP and anti-tag antibodies; data not shown). Interestingly, in contrast to our previous observations in OPCs, astrocytes (Supplementary Figure S3b) exposed to $\mathrm{TNF}-\alpha$ displayed a significant increase in $\left[\mathrm{Ca}^{2+}\right] \mathrm{m}$ transiently evoked by agonist stimulation, suggesting that a different pathway was activated than that in OPCs.

To validate the link between myelination defects and mitochondrial physiology in mixed glial co-cultures, $\left[\mathrm{Ca}^{2+}\right]_{\mathrm{m}}$ was monitored using the FRET-based probe mtD3cpv. Our analysis was restricted to cells with clear OPC-like morphology. Mitochondrial $\mathrm{Ca}^{2+}$ uptake was then stimulated by $500 \mu \mathrm{M}$ CCh, as in the aequorin experiments. As previously observed, the OPCs exposed to TNF- $\alpha$ exhibited significantly reduced $\left[\mathrm{Ca}^{2+}\right]_{\mathrm{m}}$ peaks (Supplementary Figure $\mathrm{S} 4 \mathrm{a}$ ).

To better understand how TNF- $\alpha$ affects mitochondrial $\mathrm{Ca}^{2+}$ handling, we monitored the mitochondrial network 

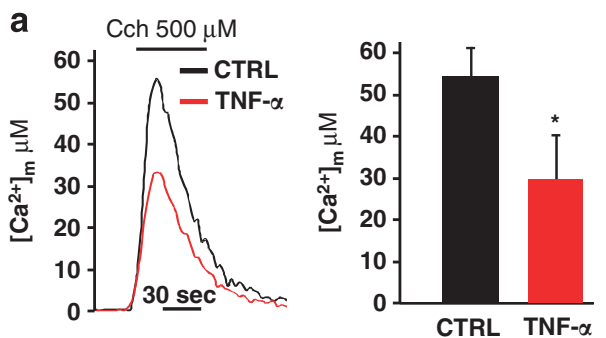

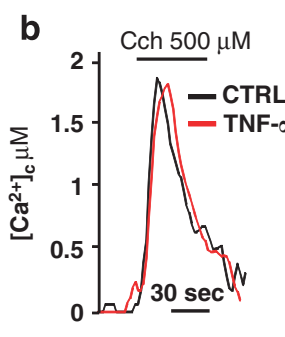

c
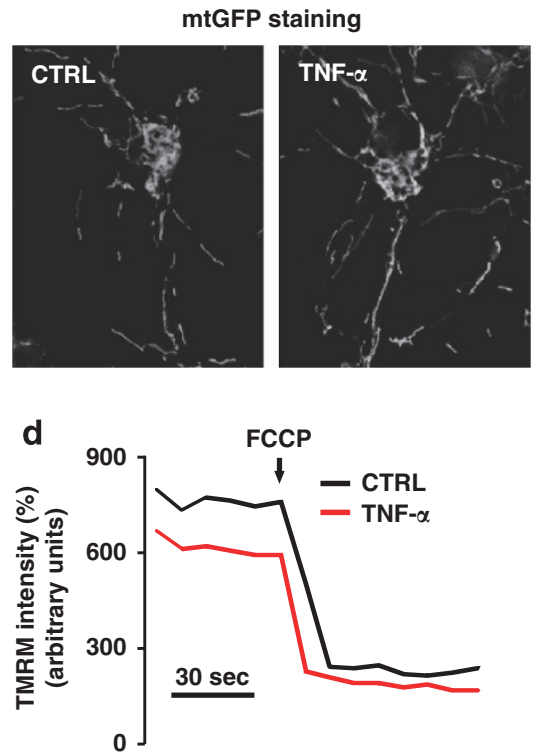

total volume
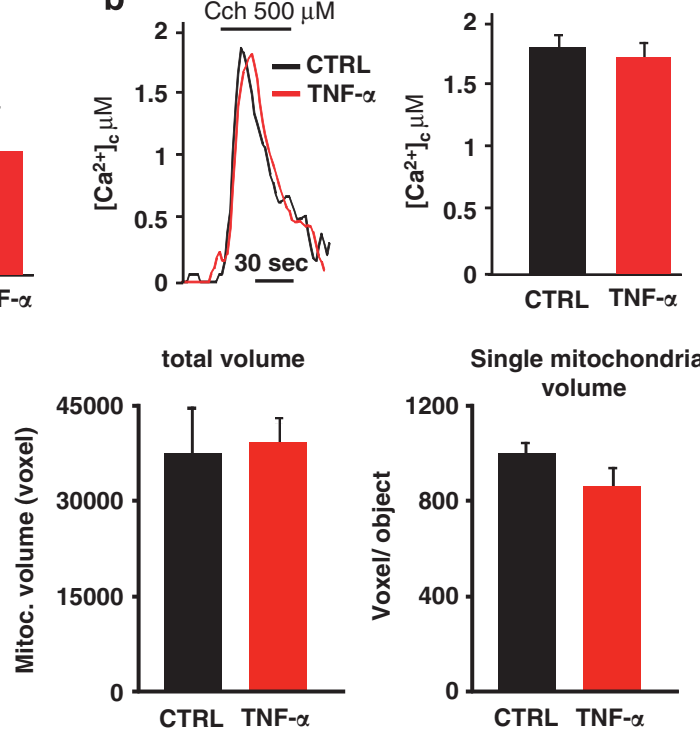

CTRL TNF- $\alpha$

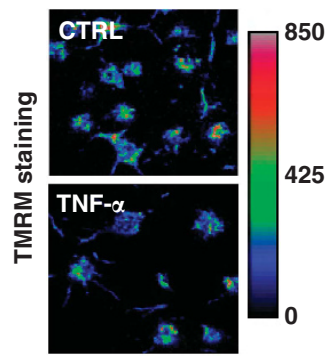

Figure 3 (a) Aequorin-based measurements of mitochondrial $\mathrm{Ca}^{2+}$ uptake (mean \pm S.E.M.) induced in OPCs by stimulation with $500 \mu \mathrm{M}$ CCh in control (CTRL; $53.64 \pm 6.73 \mu \mathrm{M})$ and TNF- $\alpha$-pretreated $(29.35 \pm 9.85 \mu \mathrm{M})$ cells. (b) Measurements of $\left[\mathrm{Ca}^{2+}\right]_{\mathrm{c}}$ based on cytAEQ in OPCs (mean \pm S.E.M.: CTRL, $1.82 \pm 0.08 \mu \mathrm{M}$; TNF- $\alpha$, $1.72 \pm 0.09 \mu \mathrm{M}$ ) after stimulation with $500 \mu \mathrm{M}$ CCh. (c) Maximum intensity projection of the mitochondrial network in OPCs expressing mtGFP. The network morphology was analyzed by measuring the total mitochondrial volume (mean \pm S.E.M.: CTRL, $40837 \pm 12213.19$ voxels; TNF- $\alpha, 43501 \pm 6463.32$ voxels) and the average mitochondrial volume (CTRL, $1021.35 \pm 183.17$ voxels; TNF- $\alpha, 922.69 \pm 183.17$ voxels). (d) $\Delta \Psi_{m}$ measurements in OPCs exposed to TNF- $\alpha$ for $24 \mathrm{~h}$ and in CTRL OPCs before and after treatment with $1 \mu \mathrm{M}$ FCCP (for baseline detection), with relative quantification (mean \pm S.E.M.: CTRL, 123.882 \pm 21.52 A.F.U.; TNF- $\alpha, 93.574 \pm 9.85$ A.F.U.). Representative pseudocolored images are shown on the right. *Significant at $P<0.05$; bars $=$ S.E.M. from at least three independent experiments

morphology and mitochondrial membrane potential $\left(\Delta \Psi_{\mathrm{m}}\right)$ under the same conditions.

Alterations in the mitochondrial structure were evaluated using digital deconvolution microscopy in OPCs infected by an adenoviral vector carrying cDNA encoding a mitochondriatargeted green fluorescent protein (GFP). ${ }^{19}$ The number and mean volume of mitochondria per cell were also determined for both TNF- $\alpha$-exposed and control oligodendrocytes. Neither the total network volume nor the single particle volume was significantly different between the different experimental conditions (Figure 3c).

$\Delta \Psi_{\mathrm{m}}$ was then monitored by confocal microscopy using the potentiometric dye tetramethylrhodamine methyl ester (TMRM). ${ }^{20}$ Cells were first treated with either $10 \mathrm{ng} / \mathrm{ml}$ TNF- $\alpha$ or vehicle for $24 \mathrm{~h}$ and then loaded with $10 \mathrm{nM}$ TMRM. The decreased fluorescence caused by maximally uncoupling concentrations of carbonyl cyanide 4-(trifluoromethoxy)phenylhydrazone (FCCP) was used to normalize the signal between different experiments and individual cells (see Materials and Methods section). A $25.4 \%$ reduction in $\Delta \Psi_{\mathrm{m}}$ was observed in the cells treated with TNF- $\alpha$, suggesting an important decrease in their respiratory chain activity or mitochondrial uncoupling (Figure 3d). Again, similar results were produced in mixed glial co-cultures (Supplementary Figure S4b).

As TMRM is a lipophilic cation, its concentration in the cytoplasm depends on the amplitude of the membrane potential across the plasma membrane. ${ }^{21}$ In turn, a decrease in the plasma membrane voltage decreases the level of cytosolic TMRM and thus the accumulation of the dye by mitochondria, even if $\Delta \Psi_{m}$ is not affected. To confirm that the reduction in the mitochondrial TMRM signal was not dependent on a change in the plasma membrane potential, we measured the cytoplasmic TMRM intensity in OPCs exposed to TNF- $\alpha$ or control treatment (by thresholding the mitochondrial TMRM signal; see Materials and Methods section). No significant differences were observed after cytokine exposure, suggesting that the difference in the TMRM signal between controls and TNF- $\alpha$-treated cells was indeed due to a decrease in $\Delta \Psi_{\mathrm{m}}$ only (Supplementary Figure S5).

It has been reported that electron leakage from the respiratory chain leads to a reduction in $\Delta \Psi_{\mathrm{m}}$ and promotes the generation of superoxide anions, which are in turn considered to be good descriptive markers of ROS production. ${ }^{22}$ 
In addition, TNF- $\alpha$ has already been shown to increase superoxide levels in leukemia cells. ${ }^{23}$ In the current study, mitochondrial superoxide anion levels in oligodendrocyte progenitors were determined using the $\mathrm{O}_{2}^{-}$-sensitive mitochondrial probe MitoSOX. We found that mitochondrial superoxide anion levels were indeed significantly increased in TNF- $\alpha$-treated OPCs compared with controls (Figure 4a).

Several reports indicate that a reduction in $\Delta \Psi_{\mathrm{m}}$ and an increase in ROS levels are most often associated with defects in complex I of mitochondrial respiratory chain. ${ }^{24,25}$ Thus, we monitored both the levels of the respiratory chain complexes and their activity. The expression profiles of the respiratory complexes were measured by western blotting with antibodies raised against representative subunits of each complex (see Materials and Methods section). No significant changes in the expression levels of complex markers were induced by TNF- $\alpha$ treatment (Figure 4b). Furthermore, we used blue native electrophoresis (with an in-gel activity assay) to monitor the activity of respiratory complex I. Interestingly, TNF- $\alpha$ treatment of OPCs caused a $22.7 \pm 5.25 \%$ decrease in the activity of complex I compared with its activity under control conditions (Figure 4c).

Finally, we investigated whether the alterations in mitochondrial functions and ROS metabolism that were caused by TNF- $\alpha$ were maintained throughout differentiation or whether differentiating OPCs underwent adaptation.

After 5 days of T3-induced differentiation, $\left[\mathrm{Ca}^{2+}\right]_{\mathrm{m}}$ was monitored in single cells using the $\mathrm{Ca}^{2+}$-sensitive, FRETbased probe mtD3cpv. Differentiating OPCs in the presence of TNF- $\alpha$ retained significantly reduced $\left[\mathrm{Ca}^{2+}\right]_{m}$ elevations in response to CCh (Figure 5a). Moreover, the MitoSOX signal was higher in treated cells compared with controls (Figure 5b), whereas the levels of the respiratory chain complexes and of ATP synthase remained unchanged throughout the differentiation process (Figure $5 c$ ).

To verify whether the impaired $\Delta \Psi_{\mathrm{m}}$ and the induced ROS were linked to TNF- $\alpha$ during its inhibition of differentiation, we compared the effects of the respiratory complex inhibitors rotenone (Rot, for complex I) and antimycin $A$ ( $A A$, for complex III) with the effects of TNF- $\alpha$ on the OPC differentiation process. Previous report indicated that inhibition of mitochondrial respiratory complex I using a selective inhibitor result in a significant inhibition of differentiation. ${ }^{4}$ OPCs were exposed to TNF- $\alpha$ at $10 \mathrm{ng} / \mathrm{ml}$, Rot at $10 \mathrm{nM}$, or AA at $100 \mathrm{nM}$ in albumin-containing medium that was refreshed every $48 \mathrm{~h}$. Having previously observed the major relevance of the proportions of progenitors and adult cells, we considered only the levels of $\mathrm{NG}_{2}{ }^{+} / \mathrm{OSP}^{+}$cells and $\mathrm{MBP}^{+} / \mathrm{OSP}^{+}$cells. The ratio of $\mathrm{NG}^{+} / \mathrm{OSP}^{+}$cells was significantly higher when cells were exposed to TNF- $\alpha$ at $10 \mathrm{ng} / \mathrm{ml}$, Rot at $10 \mathrm{nM}$, or $\mathrm{AA}$ at $100 \mathrm{nM}$ (Figure 6Ai). At the same time the proportion of $\mathrm{MBP}^{+} / \mathrm{OSP}^{+}$cells was significantly reduced (Figure 6aii). An analogous effect was induced by a submaximal dose of FCCP $(500 \mathrm{nM})$. The mild mitochondrial depolarization induced by FCCP resulted in a slight enrichment of $\mathrm{NG}_{2}^{+}$/ $\mathrm{OSP}^{+}$cells and a decrease in the proportion of $\mathrm{MBP}^{+} / \mathrm{OSP}^{+}$ cells (Supplementary Figure S6).

The modest reduction in $\Delta \Psi_{\mathrm{m}}$ and the reduced activity of respiratory chain complex I suggest that the cells could activate their energy production via glycolysis. In turn, we predicted that OPCs exposed to TNF- $\alpha$ would display increased levels of phosphorylated AMP-activated protein
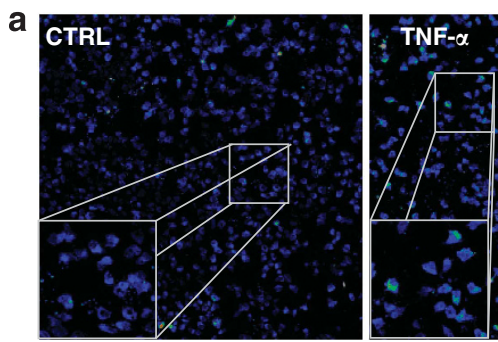

b

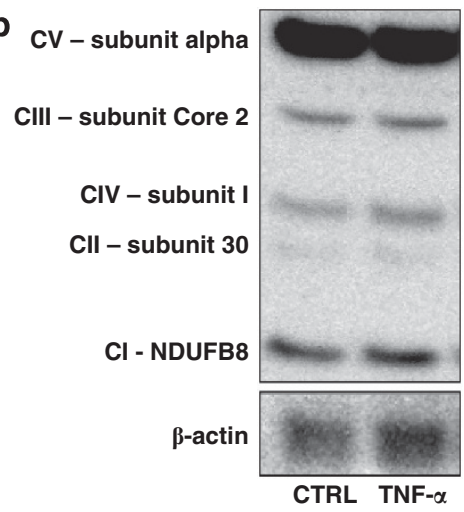

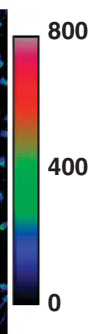

C
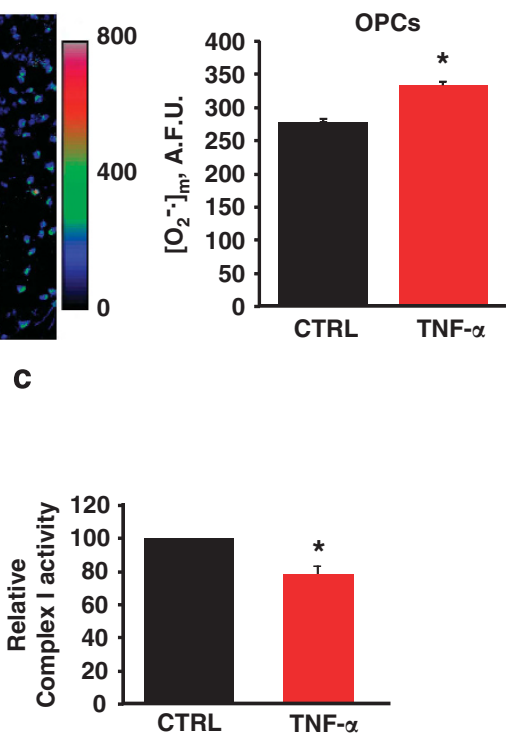

Figure 4 (a) Representative pseudocolored images of MitoSOX-stained OPCs and measurement of superoxide production in control (CTRL) cells (mean \pm S.E.M.: CTRL, $277.87 \pm 86.99$ A.F.U.; TNF- $\alpha, 333.454 \pm 101.08$ A.F.U.). (b) Expression levels of different respiratory complex subunits in CTRL and TNF- $\alpha$-treated OPCs. (c) Relative activity of respiratory complex I, measured as integrated density in a blue native gel and expressed as a percentage of the CTRL (mean \pm S.E.M.: TNF- $\alpha, 77.77 \pm 5.25$ A.U.). *Significant at $P<0.05$; bars, S.E.M. from at least three independent experiments 
a
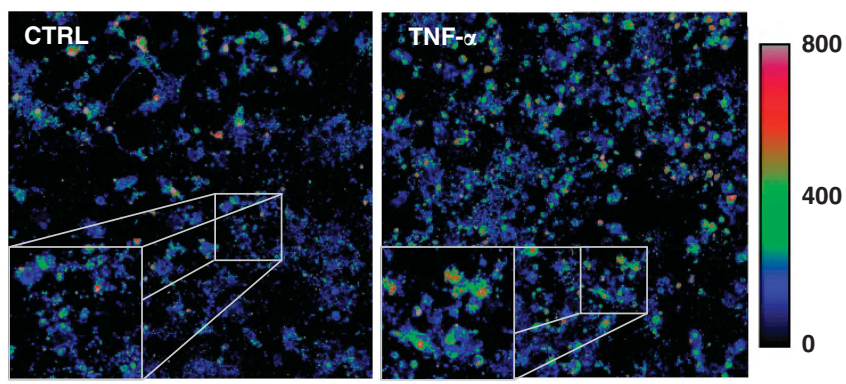

b

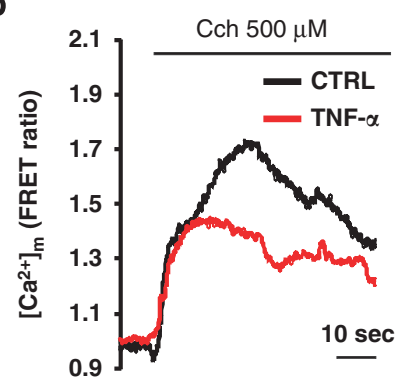

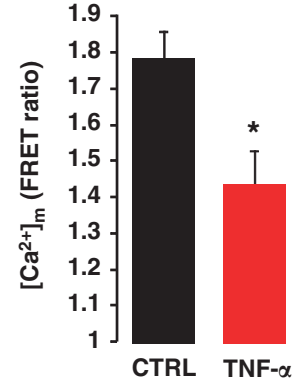

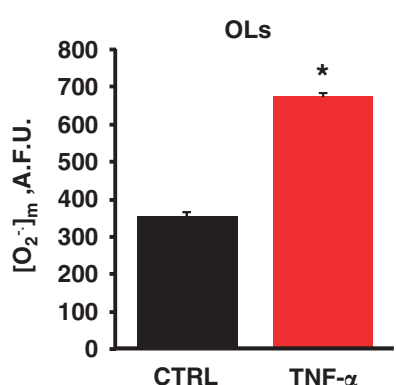

C CV-subunit alpha

CIII - subunit Core 2

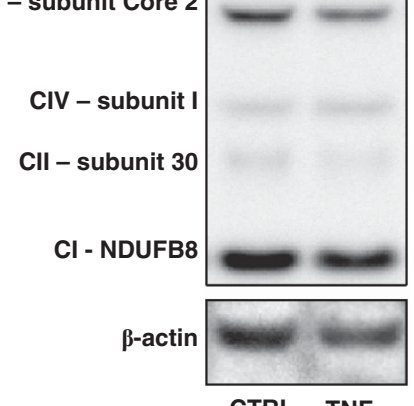

Figure 5 (a) Representative pseudocolored images of MitoSOX-stained mature OLs and measurements of superoxide production in control (CTRL) cells (mean \pm S.E.M.: CTRL, $347.88 \pm 178.6$ A.F.U.; TNF- $\alpha, 676.93 \pm 268$ A.F.U.). (b) Single-cell measurement and relative quantification of $\left[\mathrm{Ca}^{2+}\right]_{\mathrm{m}}$ in OLs expressing mtD3cpv (mean \pm S.E.M.: CTRL, $1.75 \pm 0.085$ A.F.U.; TNF- $\alpha, 1.46 \pm 0.1$ A.F.U.). (c) Expression levels of different respiratory complex subunits in CTRL and TNF- $\alpha$-treated OLs. ${ }^{*}$ Significant at $P<0.05$; bars, S.E.M. from at least three independent experiments

kinase (AMPK), which was confirmed by western blotting (Figure 6c). In addition, AMPK can inhibit the autophagy inhibitor mechanistic target of rapamycin (mTOR). ${ }^{26}$ Interestingly, the mTOR pathway is a known regulator of the oligodendrocyte differentiation process. ${ }^{27}$ Indeed OPCs exposed to TNF- $\alpha$ indeed exhibited higher conversion levels for LC3 (Figure 6d), an autophagy marker, consistent with activation of the AMPK pathway and inhibition of mTOR.

To confirm increased dependence on glycolytic metabolism in the presence of $\mathrm{TNF}-\alpha$, the respiration rate and lactate production were measured in OPCs. The respiration rate was specifically investigated by monitoring $\mathrm{O}_{2}$ consumption using nanoparticles coated with the oxygen-sensitive probe MitoXpress-Intra and measured by time-resolved fluorimetry. The use of this technique allows a sensitive measurement of oxygen consumption, even within a small cell amount, in contrast to what is required by the Clarck's electrode method. Here, the lifetime signal was inversely proportional to the $\mathrm{O}_{2}$ concentration and directly proportional to the oxygen consumption rate. The basal oxygen consumption rate was also measured for $15 \mathrm{~min}$ and then normalized to the relative minimal signal obtained by exposure to $1 \mu \mathrm{M}$ AA for $15 \mathrm{~min}$. The difference between the basal lifetime signal and the signal after AA treatment was used as an index of the respiration rate. As expected, OPCs exposed to TNF- $\alpha$ at $10 \mathrm{ng} / \mathrm{ml}$ displayed a $22 \%$ reduction in their oxygen consumption rates (Figure 6d).

Finally, lactate production was supposed to be augmented in OPCs exposed to the cytokine. Lactate levels were investigated by measuring the amount of lactate released into the medium using a colorimetric kit. As expected, OPCs exposed to fresh medium containing TNF- $\alpha$ at $10 \mathrm{ng} / \mathrm{ml}$ for $24 \mathrm{~h}$ displayed an increased lactate concentration compared with controls (Figure 6e).

\section{Discussion}

Myelin degeneration and axon remyelination failure in oligodendrocytes, which are associated with differentiation dysfunction, are pathological hallmarks of MS. Several lines of evidence indicate that TNF- $\alpha$ is one of the most relevant cytokines involved in the pathogenesis of this disease $e^{7,8,28-30}$ and mimics the effect of the disease on oligodendrocytes in vitro by promoting both cell death and impairment of the differentiation process. ${ }^{12,31}$ In the present study, we confirmed that exposure to low concentrations of TNF- $\alpha$ $(10 \mathrm{ng} / \mathrm{ml})$ impairs OPC differentiation, causing an accumulation of early progenitors and reducing the relative amounts of differentiating oligodendrocytes in vitro. This phenomenon was not accompanied by a significant induction of apoptosis, which occurred only when the TNF- $\alpha$ concentration was increased to $100 \mathrm{ng} / \mathrm{ml}$.

The inhibition of OPC differentiation resulted in an impairment of myelination capacity, as shown in mixed glial co-culture experiments. These data are consistent with the accumulation of progenitor cells at the lesion sites observed in MS patients ${ }^{32}$ and suggest that failed remyelination is a consequence of the inhibition of differentiation. ${ }^{33,34}$ 
a
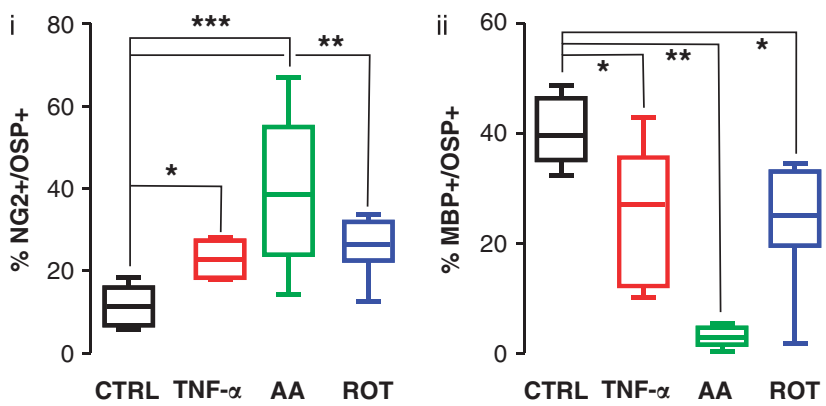

b

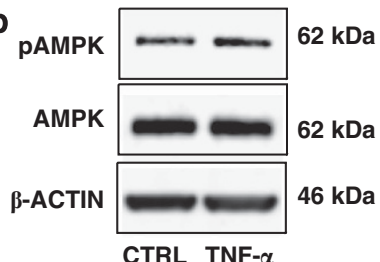

d

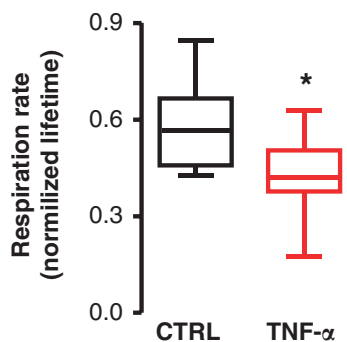

C
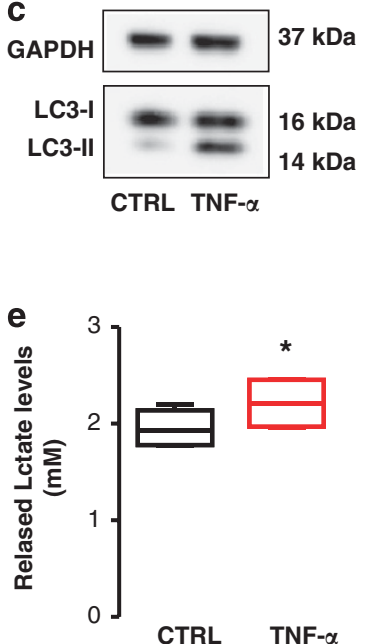

Figure 6 Differentiation assay in OPCs pretreated with control (CTRL) treatment, $10 \mathrm{ng} / \mathrm{ml}$ TNF- $\alpha, 10 \mathrm{nM}$ Rot, or $100 \mathrm{nM} \mathrm{AA}$ and then treated with the thyroid hormone T3 for 5 days. The proportions of cells expressing different markers were counted among the cells that were positive for the O4 OSP. Percentages (mean \pm S.E.M.) of NG2 ${ }^{+}$cells (progenitors, a(i)): CTRL, $10.9 \pm 1.638 \%$; TNF- $\alpha$, $22.325 \pm 8.46 \% ; \quad$ AA, $38.72 \pm 8.46 \%$; Rot, $26.13 \pm 2.47 \%$. Percentages (mean \pm S.E.M.) of $\mathrm{MBP}^{+}$cells (adult, a(ii)): CTRL $39.57 \pm 2.17 \%$; TNF- $\alpha$, $27.4 \pm 4.62 \%$; AA, $3.18 \pm 0.63 \%$; Rot, $25.36 \pm 3.84 \%$. *Significant at $P<0.05$, ** significant at $P<0.01$, and ${ }^{* * *}$ significant at $P<0.005$; line, mean; bars, maximum and minimum values. The boxes envelop the 25 th to the 75 th percentile of the assayed population, including $n=3$ independent cultures. (b) Expression levels of AMPK alpha and its phosphorylated form, as monitored by western blotting in control (CTRL) and TNF- $\alpha$-exposed OPCs. (c) LC3 conversion, as monitored by western blotting in CTRL and TNF- $\alpha$-exposed OPCs. (d) Respiration rate in CTRL OPCs and OPCs exposed to TNF- $\alpha$ at $10 \mathrm{ng} / \mathrm{ml}$ and monitored by time-resolved fluorimetry using the $\mathrm{O}_{2}$-sensitive probe MitoXpress-Intra. The values are expressed as normalized lifetimes (mean \pm S.E.M.: CTRL, $0.56 \pm 0.038$; TNF- $\alpha 0.42 \pm 0.044$ ). *Significant at $P<0.05$; line, mean; bars, maximum and minimum values. The boxes envelop the 25th to the 75th percentile of the assayed population, including $n=3$ independent experiments. (e) Lactate concentration in media from CTRL OPCs and OPCs exposed to TNF- $\alpha$ at $10 \mathrm{ng} / \mathrm{ml}$ and monitored by spectroscopy of the colorimetric conversion of a lactate-sensitive substrate (mean \pm S.E.M: CTRL, $1.93 \pm 0.098 \mathrm{mM}$; TNF- $\alpha, 2.21 \pm 0.13 \mathrm{mM}$ ). ${ }^{*}$ Significant at $P<0.05$; line, mean bars, maximum and minimum values. The boxes envelop the 25th to the 75th percentile of the assayed population, and each dot represents an assayed culture, with $n=4$ independent experiments

Oligodendrocyte differentiation and myelination have been proposed to be highly energy-consuming events that require functional mitochondria both in vitro and in vivo, in mouse models. ${ }^{4,5,35}$ Furthermore, mitochondrial dysfunction has been associated with the development of MS and with impairments in oligodendrocyte differentiation. ${ }^{4,36}$

We hypothesized that if TNF- $\alpha$ could condition mitochondrial physiology, this mechanism could be underlying TNF- $\alpha$-induced differentiation inhibition. Altered mitochondrial $\mathrm{Ca}^{2+}$ homeostasis is often an initial reporter of changes in mitochondrial physiology. The results indicated that a decrease in mitochondrial $\mathrm{Ca}^{2+}$ uptake occurred in TNF- $\alpha$-treated cultures and that this event was not accompanied by alterations in cytoplasmic $\mathrm{Ca}^{2+}$ peaks. This finding suggested that the alterations in $\left[\mathrm{Ca}^{2+}\right]_{\mathrm{m}}$ signals were not due to a general perturbation of intracellular $\mathrm{Ca}^{2+}$ homeostasis but were instead specific mitochondrial defects. Interestingly, no significant alterations in $\left[\mathrm{Ca}^{2+}\right]_{\mathrm{m}}$ were detected after exposure to IFN- $\gamma$, indicating that the observed impairment of mitochondrial activity is not a general feature of proinflammatory cytokines but is typical of TNF- $\alpha$.

Mitochondrial $\mathrm{Ca}^{2+}$ uptake occurs through the mitochondrial $\mathrm{Ca}^{2+}$ uniporter complex (extensively reviewed in Marchi and Pinton ${ }^{37}$ ), utilizing the $\Delta \Psi_{\mathrm{m}}$, as driving force; thus a decrease in this parameter reduces the accumulation of $\mathrm{Ca}^{2+}$ by the organelle. Moreover, once in the mitochondria, $\mathrm{Ca}^{2+}$ rapidly diffuses from the main uptake sites (the sites of contact with the endoplasmic reticulum) to the entire network. Owing to these features, both variations in $\Delta \Psi_{\mathrm{m}}$ and changes in mitochondrial network interconnections can affect mitochondrial $\mathrm{Ca}^{2+}$ uptake. Although the mitochondria of treated cells did not significantly change in size or number, they displayed significantly lower $\Delta \Psi_{\mathrm{m}}$, suggesting that this reduced $\Delta \Psi_{\mathrm{m}}$ is responsible for the reduced $\mathrm{Ca}^{2+}$ accumulation by the organelle.

In leukemia cells, TNF- $\alpha$ has been shown to induce cell death by selectively promoting ROS generation via respiratory complex I. ${ }^{23}$ In contrast, in OPCs, at the TNF- $\alpha$ concentrations analyzed, no cell death was observed, and there was significantly higher MitoSOX staining than in controls. This phenomenon was accompanied by a significant decrease in the activity of complex I compared with its activity under control conditions. Interestingly, this decrease was not accompanied by changes in the levels of the NDUFB8 subunit of complex I (or of any other OXPHOS component). These results suggest that $\mathrm{TNF}-\alpha$ treatment impairs respiratory complex I in OPCs, which results in reduced $\Delta \Psi_{\mathrm{m}}$ and increased $\left[\mathrm{Ca}^{2+}\right]_{\mathrm{m}}$ upon stimulation and in increased superoxide levels.

Our results are consistent with the observations that patients with mutations in complex I have a 100-fold increased risk of developing $\mathrm{MS}^{38,39}$ and that low doses of Rot (an inhibitor of complex I), which do not affect cell viability or ATP synthesis, inhibit OPC differentiation. ${ }^{4}$

If impaired mitochondrial functions are fundamental to the differentiation blockade caused by TNF- $\alpha$, this dysfunction should be maintained during the differentiation process. Indeed, OPCs exposed to TNF- $\alpha$ and subsequently induced to differentiate by T3 exhibited higher MitoSOX staining and lower $\left[\mathrm{Ca}^{2+}\right]_{\mathrm{m}}$ peaks in response to $\mathrm{CCh}$.

In support of this model, the mitochondrial poisons Rot and AA were able to impair the differentiation process in OPC, confirming the findings of a previous study. ${ }^{4}$ Rot and AA are also able to inhibit respiratory complexes I and III, respectively, causing a dramatic reduction in $\Delta \Psi_{\mathrm{m}}$ and increase in ROS production. ${ }^{10,40}$ Interestingly, in the present study, Rot 
induced impairment that was most comparable with the impairment induced by TNF- $\alpha$. This observation is consistent with the reduced complex I activity observed in OPCs (in this study) and in leukemic cells ${ }^{23}$ exposed to TNF- $\alpha$, strongly suggesting that respiratory complex $\mathrm{I}$ is the site of entry for the TNF- $\alpha$ signaling pathway's effects on mitochondrial physiology.

Interestingly, exposure to the mitochondrial uncoupler FCCP, which is usually associated with an inhibition of ROS production, also resulted in impairment of OPC differentiation, although to a lesser extent compared with Rot and AA. This finding suggests that impaired mitochondrial physiology is sufficient to generate a delay in OPC differentiation and implies a possible explanation for the observed association between genetic variants of UCPs in two types of demyelinating disorders: MS and leukoaraiosis. ${ }^{41-44}$ In addition, a concomitant increase in ROS would worsen the impairment of differentiation, explaining the correlation between increased ROS levels and the severity of the demyelinating disorder. ${ }^{38,39}$

On the basis of these results, the cells exposed to TNF- $\alpha$ were expected to exhibit metabolic impairments. To test this hypothesis, we verified the levels and activity of the metabolic sensor AMPK. This protein is relevant to the regulation of cellular energy metabolism and is able to sense mitochondrial activity or ROS levels to promote glycolysis. ${ }^{45}$ Interestingly, cells treated with TNF- $\alpha$ displayed higher levels of phosphorylated forms of the alpha subunit of AMPK. AMPK has already been shown to be able to increase the glycolytic flux during mitochondrial stress. ${ }^{45}$ In the current study, OPCs exposed to TNF- $\alpha$ displayed both reduced $\mathrm{O}_{2}$ consumption and increased lactate production, indicating an increased in their dependence on glycolysis. In addition, AMPK is a well-known inhibitor of the mTOR pathway, ${ }^{46}$ a negative regulator of autophagy. ${ }^{47}$ To confirm that the observed AMPK activation was due to TNF- $\alpha$, we verified LC3 conversion (an autophagy marker) as readout of mTOR activity. In agreement with our hypothesis, OPCs exposed to TNF- $\alpha$ displayed higher LC3 conversion, indicating that mTOR activity was indeed inhibited.

Because the mTOR pathway is a central promoter of OPC differentiation $^{27}$ and given the results of the present study, we speculate that the inhibition of differentiation caused by TNF- $\alpha$ is mediated by increased levels of AMPK and greater AMPK activation.

Overall, our data indicate that TNF- $\alpha$ promotes mitochondrial dysfunction in OPCs, leading to increased activity of AMPK and in turn to inhibition of mTOR. This sequence of events eventually leads to a blockade of the differentiation of OPCs and to reduced myelination of axons in vitro. These findings provide new insights that may aid in the understanding of the role played by the inflammatory response (and by TNF- $\alpha$ in particular) in remyelination failure in MS.

\footnotetext{
Materials and Methods

Oligodendrocyte progenitor cultures generation. Primary OPC cultures were prepared following the protocol of Chen et al. ${ }^{48}$ This method involves the generation of a population of OPCs on a layer of astrocytes, originating from mixed glial cultures from P1-P2 rat pups. For the separation of the two types of primary cell cultures, after 10 days of growth in DMEM with $20 \% \mathrm{FBS}$, the entire preparation was exposed to sheer forces generated by shaking on an
}

orbital shaker at $190 \mathrm{rpm}$. The oligodendrocyte progenitors were then collected and separated from residual microglia and astrocytes by incubation for $1 \mathrm{~h}$ on an uncoated Petri dish and then filtered through cell strainers with $40 \mu \mathrm{M}$ pores. Next, the cells were seeded on coverslips of different dimensions, according to the planned experiment, and maintained in culture for 7 days in chemically defined medium composed of DMEM (EuroClone, Milan, Italy), $4 \mathrm{mM}$ L-glutamine (EuroClone), $1 \mathrm{mM}$ sodium pyruvate (Sigma Aldrich, St. Louis, MO, USA), $0.1 \%$ BSA (Sigma Aldrich), $50 \mathrm{mg} / \mathrm{ml}$ apo-transferrin (Sigma Aldrich), $5 \mathrm{mg} / \mathrm{ml}$ insulin (Sigma Aldrich), $30 \mathrm{nM}$ sodium selenite (Sigma Aldrich), $10 \mathrm{nM}$ D-biotin (Sigma Aldrich), $10 \mathrm{nM}$ hydrocortisone (Sigma Aldrich), $10 \mathrm{ng} / \mathrm{ml}$ PDGF-AA (PeproTech, Rocky Hill, NJ, USA), and $10 \mathrm{ng} / \mathrm{ml}$ bFGF (Merck Millipore, Billerica, MA, USA).

Adenovirus infection. OPCs grown for 7 days were exposed to adenoviral vectors carrying different cDNAs (mtAEQ; cytAEQ; mitochondrial GFP, or mtGFP; $\mathrm{mtD} 3 \mathrm{cpv}$; or $4 \mathrm{mtD} 3 \mathrm{cpv}$ ), with $1 \mu \mathrm{l}$ diluted into $500 \mu \mathrm{l}$ of complete medium. After $16 \mathrm{~h}$ of incubation, the medium was replaced with fresh medium, and reporter expression was monitored after $24 \mathrm{~h}$.

Aequorin measurements. All aequorin measurements were performed as previously described. ${ }^{16}$ OPCs grown on $13 \mathrm{~mm}$ round glass coverslips were infected with $1 \mu \mathrm{l}$ of adenovirus carrying aequorin cDNA (cytAEQ or mtAEQ). After $36 \mathrm{~h}$, the cells were reconstituted and placed in a perfused thermostated chamber in which the light signal was collected using a purpose-built luminometer and converted into $\left[\mathrm{Ca}^{2+}\right]$ values. The cells transfected with cytAEQ or $\mathrm{mtAEQ}$ were reconstituted with coelenterazine (Synchem, Felsberg/Altenburg, Germany) for $2 \mathrm{~h}$ in KRB supplemented with $1 \mathrm{mM} \mathrm{CaCl}$. All aequorin measurements were performed in $1 \mathrm{mM} \mathrm{Ca}^{2+} / \mathrm{KRB}$ (for both cytAEQ and $\mathrm{mtAEQ}$ ). An agonist was added to the same medium, as specified in the figures. The experiments were finished by lysing the cells with $100 \mathrm{mM}$ digitonin in a hypotonic, $\mathrm{Ca}^{2+}$-rich solution ( $10 \mathrm{mM} \mathrm{CaCl}_{2}$ in $\mathrm{H}_{2} \mathrm{O}$ ).

Western blotting. Cells were lysed using RIPA buffer $(50 \mathrm{mM}$ Tris- $\mathrm{HCl}$ pH 7.8, $150 \mathrm{mM} \mathrm{NaCl}, 1 \%$ IGEPAL CA-630, 0.5\% sodium deoxycholate, $0.1 \%$ SDS, and $1 \mathrm{mM}$ DTT) supplemented with protease and phosphatase inhibitors $\left(2 \mathrm{mM} \mathrm{Na}_{3} \mathrm{VO}_{4}, 2 \mathrm{mM} \mathrm{NaF}, 1 \mathrm{mM}\right.$ PMSF, and protease inhibitor cocktail) following three freeze-thaw cycles. Next, $30 \mu \mathrm{g}$ of protein was separated on a NuPAGE Novex 4-12\% Bis-Tris precast gel (Life Technologies, Carlsbad, CA, USA) and transferred to PVDF membranes (Bio-Rad Laboratories, Hercules, CA, USA) for standard western blotting. Immunoblotting of the membranes was performed using the following primary antibodies: antiNG2 (1: 1000; Santa Cruz Biotechnology, Santa Cruz, CA, USA), anti-MBP (1:1000; Abcam, Cambridge, UK), anti-04 (1:1000; Abcam), anti-cPARP (1:500; Cell Signaling, Beverly, MA, USA), anti- $\beta$-tubulin III (1:1000; Sigma), anti-panVDAC (1:5000; Abcam), anti-actin (1:3000; Santa Cruz), anti-OxPhos (1:1000; MitoSciences, Eugene, OR, USA), anti-GAPDH $(1: 6000$; Cell Signaling), anti-AMPK (1:1000; Cell Signaling), anti-pAMPK antibody (1:1000; Cell Signaling), and anti-LC3 (1:2000; Sigma Aldrich).

Isotype-matched, horseradish-peroxidase conjugated secondary antibodies were then used, followed by detection with chemiluminescence (Perkin Elmer, Waltham, MA, USA).

Blue native electrophoresis. Cell pellets were solubilized in $1.5 \mathrm{M}$ aminocaproic acid, $50 \mathrm{mM}$ Bis-Tris- $\mathrm{HCl}(\mathrm{pH} 7.0)$, and $1 \%$ dodecylmaltoside. These samples were incubated on ice for 20-30 min and centrifuged at $15000 \times \mathrm{g}$ for $15 \mathrm{~min}$ to remove unsolubilized material. The protein concentration in the supernatant was determined according to Bradford ${ }^{49}$ using a Bio-Rad protein estimation kit (Bio-Rad Laboratories). Samples of the supernatant, containing $60 \mu \mathrm{g}$ of protein each, were then combined with $1 \mu \mathrm{l}$ of $5 \%$ Coomassie brilliant blue and separated on a large $(1 \mathrm{~mm} \times 16 \mathrm{~cm} \times 20 \mathrm{~cm}) \quad 5-12 \%$ gradient acrylamide gel. Identification of the activity of respiratory chain complex I in slices of this acrylamide separation gel was performed with the use of an in-gel activity assay.

In-gel activity assay. To visualize complex I activity, the gel was incubated at $35^{\circ} \mathrm{C}$ in $3 \mathrm{mM}$ Tris-HCl (pH 7.4) buffer supplemented with $60 \mu \mathrm{M} \mathrm{NADH}$ and $245 \mu \mathrm{M}$ nitrotetrazolium blue (NBT). The incubation was terminated when the characteristic band for complex I became visible (the process takes $\sim 18 \mathrm{~h}$ for this type of sample). 
Mitochondrial morphology analysis. OPCs were seeded at 50000 or 20000 cells per $25 \mathrm{~mm}$ coverslip, grown for $24 \mathrm{~h}$, and then infected with a mitochondria-targeted GFP inserted into an adenoviral vector (Ad-mtGFP). After $36 \mathrm{~h}$ of expression, the cells were treated as described and then imaged with a LiveScan Swept Field Confocal Microscope (Nikon Instruments Inc., Melville, NY, USA) equipped with a $\times 60$ oil immersion objective (N.A. 1.4; Nikon Instruments) and an Andor DU885 EM-CCD camera (Andor Technology, Belfast, UK). After the coverslips were placed in an incubation chamber with controlled temperature, $\mathrm{CO}_{2}$, and humidity, $\mathrm{z}$-stacks were acquired on 21 planes separated by a $0.6 \mu \mathrm{m}$ distance to allow acquisition of the entire cell. GFP ${ }^{+}$objects were quantified with the '3D object counter' plug-in of open-source Fiji software (freely available at http://fiji.sc/), whereas 3D representations were obtained with the '3D Viewer' plug-in.

Immunostaining. Cells were fixed with $3.7 \%$ formaldehyde in PBS for $20 \mathrm{~min}$, washed three times with PBS, and then incubated for $10 \mathrm{~min}$ in PBS supplemented with $50 \mathrm{mM} \mathrm{NH}{ }_{4} \mathrm{Cl}$. Permeabilization of cell membranes was achieved with a 5 min incubation with $0.1 \%$ Triton X-100 in PBS, followed by a $1 \mathrm{~h}$ wash with $2 \%$ nonfat dry milk in PBS. The cells were then incubated $\mathrm{O} / \mathrm{N}$ at $37^{\circ} \mathrm{C}$ in a wet chamber with the following antibodies: mouse anti-NG2 (Santa Cruz), mouse anti-O4 (Sigma Aldrich), mouse anti-MBP (Sigma Aldrich), and rabbit antiOSP (Abcam), diluted at 1:100. Staining was performed with the secondary antibodies Alexa 488 goat anti-mouse for NG2, O4, and MBP and Alexa 594 goat anti-rabbit for OSP. After incubation with each antibody, the cells were washed four times with PBS and then assessed by automated cell count.

TUNEL assay. OPCs were seeded on 96-well plates with glass bottoms. After 7 days of culture, the cells were exposed to TNF- $\alpha$, as previously described. At the end of the treatment, the OPCs were labeled using the Click-iT TUNEL Alexa Fluor 488 Imaging Assay, following the manufacturer's instructions. An automated cell count was then performed (Life Technologies).

Automated cell count. Cultured OPCs were seeded on 96-well plates with glass bottoms. After 7 days of culture, the cells were induced to differentiate by replacing PDGF-AA and bFGF with $15 \mathrm{nM}$ T3 for 5 days. Immunostained was then performed as previously described. Images were acquired using an Olympus Scan^R station (Olympus, Munster, Germany) with laser-based hardware and an image-based automatic autofocus. In total, 80 fields were acquired for each well using $\times 20$ magnification (N.A. 0.75 ), and different fluorophores were excited by an MT20 illumination system. The excitation filters that were used were $377 / 50,498 / 20$, and $595 / 30$ for DAPI, FITC, and TRITC, respectively, allowing the shortest exposure time possible ( $5 \mathrm{~ms}$ for DAPI, $20-50 \mathrm{~ms}$ for FITC, and 50$150 \mathrm{~ms}$ for TRITC). Images were collected using an Orca-05G2 at full frame, without binning. The cells were then scored and counted using the Scan $\wedge$ R analysis software.

Quantification of $\Delta \Psi \mathbf{m}$. Cells were loaded with $1 \mathrm{nM}$ TMRM (Life Technologies) in Krebs-Ringer buffer supplemented with $250 \mu \mathrm{M}$ sulfinpyrazone. The cells were then placed in a humidified chamber at $37^{\circ} \mathrm{C}$ and imaged with a LiveScan Swept Field Confocal Microscope (Nikon Instruments) equipped with a $\times 60$ oil immersion objective (N.A. 1.4; Nikon Instruments) and an Andor DU885 EM-CCD camera (Andor Technology). The signal was collected as total emission $>570 \mathrm{~nm}$. TMRM fluorescence was analyzed using the NIS-Elements software package (Nikon Instruments).

FRET-based measurements of mitochondrial $\mathrm{Ca}^{2+}$. Single-cell measurements of mitochondrial $\mathrm{Ca}^{2+}$ were performed using OPCs, OLs, or mixed glial cells transfected with $4 \mathrm{mtD} 3 \mathrm{cpv}$. After $36 \mathrm{~h}$, the cells were imaged on a Zeiss Axiovert $200 \mathrm{M}$ microscope with a cooled CCD camera (Photometrics, Tucson, AZ, USA) equipped with a C-Apochromat 40X/1.2 W CORR objective and controlled by MetaFluor 7.0 software (Molecular Devices, Sunnyvale, CA, USA). Emission ratio imaging of the $4 \mathrm{mtD} 3 \mathrm{cpv}$ was accomplished using a $436 \mathrm{DF} 20$ excitation filter, a $450 \mathrm{~nm}$ dichroic mirror, and two emission filters (475/40 for ECFP and 535/25 for citrine) controlled by a Lambda 10-2 filter changer (Sutter Instruments, Novato, CA, USA). The fluorescence images were then background corrected. The exposure times were typically $100-200 \mathrm{~ms}$, and images were collected every 5-15 s.

ROS measurements. Mitochondrial $\mathrm{O}_{2}^{-}$was estimated fluorimetrically using the mitochondrial superoxide-sensitive reagent MitoSOX (Life Technologies).
OPCs grown on $25 \mathrm{~mm}$ coverslips were stained with $5 \mu \mathrm{M}$ MitoSOX and then imaged with a LiveScan Swept Field Confocal Microscope (Nikon Instruments) equipped with a $\times 60$ oil immersion objective (N.A. 1.4; Nikon Instruments) and an Andor DU885 EM-CCD camera (Andor Technology). The coverslips were placed in an incubated chamber with controlled temperature, $\mathrm{CO}_{2}$, and humidity. Fluorescence intensity was quantified using the open-source software Fiji, available at http://fiji.sc/Fiji.

$\mathrm{O}_{2}$ consumption assay. OPCs were grown to full confluence in chemically defined, supplemented medium on cell culture-treated, black 96-well optical plates (Greiner Bio-One, Monroe, NC, USA). The seeding medium was replaced with $100 \mu \mathrm{l}$ of normal growth medium containing the MitoXpress-Intra probe at $10 \mu \mathrm{g} /$ $\mathrm{ml}$, and the cells were cultured overnight. Prior to measurement, the medium was replaced with $150 \mu$ l of pre-warmed medium. Intracellular $\mathrm{O}_{2}$ was then measured using the time-resolved fluorescence mode of the Victor3 plate reader (Perkin Elmer, Boston, MA, USA). In particular, excitation was performed at $340 \mathrm{~nm}$, and emission was collected at $670 \mathrm{~nm}$. Phosphorescent intensities were measured at delay times of 30 and $70 \mu \mathrm{s}$ (with a $30 \mu \mathrm{s}$ window time). The ratio of these intensities was subsequently converted into phosphorescent lifetimes.

Lactate assay. OPCs were grown to full confluence in chemically defined, supplemented medium on cell-culture treated 96-well plates (Greiner Bio-One) and conditioned with TNF- $\alpha$ or vehicle. After $24 \mathrm{~h}$, the amount of released lactate was determined using a colorimetric L-Lactate Assay Kit (Abcam) according to the manufacturer's protocol.

Myelination assay. This assay was developed according to a previously published protocol. ${ }^{14}$ In particular, OPCs were obtained as described above, and neurons were co-cultured on a feeder layer of cultured astrocytes for 4-7 days. Before the experiments, the OPCs were treated with $10 \mathrm{ng} / \mathrm{ml} \mathrm{TNF}-\alpha$, and after $24 \mathrm{~h}$, the cells were plated on the neuron/astrocyte co-culture. After 4-6 days, the cells were fixed and immunostained for MBP and $\beta$-tubulin III, as described above, and analyzed using a LiveScan Swept Field Confocal Microscope (Nikon Instruments) equipped with a $\times 60$ oil immersion objective (N.A. 1.4, Nikon Instruments) and an Andor DU885 EM-CCD camera (Andor Technology). The extent of myelination was then determined based on areas of colocalization using a custom-made macro and Fiji software.

Chemicals. Rat IFN- $\gamma$, rat TNF- $\alpha$, Rotenone, Antimycin A, and FCCP were obtained from Sigma-Aldrich.

Statistical analysis. Unless otherwise indicated, assays were independently performed in triplicate, which yielded comparable results. The differentiation assay data were analyzed with Prism 5 (GraphPad Software, Inc., La Jolla, CA, USA), and these data are presented as a box-and-whiskers plot.

The data that are presented as the mean \pm S.E.M. were analyzed using Microsoft Excel (Microsoft Co., Redmond, USA). Statistical significance was determined by two-tailed paired Student's $t$-tests for differentiation assays and by two-tailed unpaired Student's $t$-tests with independent variances for all other assays, and $P$-values $<0.05$ were considered statistically significant.

\section{Conflict of Interest}

The authors declare no conflict of interest.

Acknowledgements. We would like to thank Dr. Lara Bighi and Augusto Bevilacqua for their extraordinary help. This research was supported by Fondazione Italiana Sclerosi Multipla (FISM), with fellowships to AB (2010/B/1) and to SP (Cod. 2012/B/11) and with the grant FISM 2008/R/18 to PP. AR and CG are supported by the Italian Ministry of Health and Associazione Italiana per la Ricerca sul Cancro (AIRC), respectively. JMS, JD, and MRW are supported by a grant from the Polish National Science Centre, UMO-2011/01/M/NZ3/02128, BIO-IMAGing in Research Innovation and Education (FP7-REGPOT-2010-1). JMS is also supported by a Ph.D. fellowship from the Foundation for Polish Science (FNP), UE, European Regional Development Fund and Operational Programme 'Innovative Economy'. TP is supported by the Italian Ministry of Education (PRIN and FIRB projects) and the CNR project 'Aging'. PP is financed by AIRC; Telethon (GGP11139B); the Italian Ministry of Education, University and Research; and the Italian Ministry of Health. 
1. Ghafourifar P, Mousavizadeh K, Parihar MS, Nazarewicz RR, Parihar A, Zenebe WJ. Mitochondria in multiple sclerosis. Front Biosci 2008; 13: 3116-3126.

2. Bonora M, Patergnani S, Rimessi A, De Marchi E, Suski JM, Bononi A et al. ATP synthesis and storage. Purinergic Signal 2012; 8: 343-357.

3. Marchi S, Giorgi C, Suski JM, Agnoletto C, Bononi A, Bonora M et al. Mitochondria-ros crosstalk in the control of cell death and aging. J Signal Transduct 2012; 2012: 329635.

4. Schoenfeld $\mathrm{R}$ et al. Oligodendroglial differentiation induces mitochondrial genes and inhibition of mitochondrial function represses oligodendroglial differentiation. Mitochondrion 2010; 10: 143-150.

5. Rinholm JE, Hamilton NB, Kessaris N, Richardson WD, Bergersen LH, Attwell D. Regulation of oligodendrocyte development and myelination by glucose and lactate. J Neurosci 2011; 31: 538-548.

6. Benarroch EE. Oligodendrocytes: susceptibility to injury and involvement in neurologic disease. Neurology 2009; 72: 1779-1785.

7. Selmaj K, Raine CS, Cannella B, Brosnan CF. Identification of lymphotoxin and tumor necrosis factor in multiple sclerosis lesions. J Clin Invest 1991; 87: 949-954.

8. Musabak U, Demirkaya S, Genc G, llikci RS, Odabasi Z. Serum adiponectin, TNF-alpha, IL-12p70, and IL-13 levels in multiple sclerosis and the effects of different therapy regimens. Neuroimmunomodulation 2011; 18: 57-66.

9. Vladić A, Horvat G, Vukadin S, Sučić Z, Šimaga Š. Cerebrospinal fluid and serum protein levels of tumour necrosis factor-alpha (TNF- $\alpha$ ), interleukin-6 (IL-6) and soluble interleukin-6 receptor (sIL-6R gp80) in multiple sclerosis patients. Cytokine 2002; 20: 86-89.

10. Koopman WJH, Nijtmans LG, Dieteren CE, Roestenberg P, Valsecchi F, Smeitink JA et al. Mammalian mitochondrial complex I: biogenesis, regulation, and reactive oxygen species generation. Antioxid Redox Signal 2010; 12: 1431-1470.

11. Chen $\mathrm{Y}$, Balasubramaniyan V, Peng J, Hurlock EC, Tallquist M, Li J et al. Isolation and culture of rat and mouse oligodendrocyte precursor cells. Nat Protoc 2007; 2: 1044-1051.

12. Zhang $X$, Haaf M, Todorich B, Grosstephan E, Schieremberg H, Surguladze N et al. Cytokine toxicity to oligodendrocyte precursors is mediated by iron. Glia 2005; $\mathbf{5 2}$ : 199-208.

13. Agresti C, Meomartini ME, Amadio S, Ambrosini E, Volonté C, Aloisi F et al. ATP regulates oligodendrocyte progenitor migration, proliferation, and differentiation: involvement of metabotropic P2 receptors. Brain Res Brain Res Rev 2005; 48: 157-165.

14. Itoh K. Culture of oligodendrocytes precursor cells (NG2 + /01-) and oligodendrocytes (NG2- /01 + ) from embryonic rat cerebrum. Brain Res Protoc 2002; 10: 23-30.

15. Pinton $P$, Rimessi A, Romagnoli A, Prandini A, Rizzuto R. Biosensors for the detection of calcium and pH. Methods Cell Biol 2007; 80: 297-325.

16. Bonora M, Giorgi C, Bononi A, Marchi S, Patergnani S, Rimessi A et al. Subcellular calcium measurements in mammalian cells using jellyfish photoprotein aequorin-based probes. Nat Protoc 2013; 8: 2105-2118.

17. Palmer AE, Tsien RY. Measuring calcium signaling using genetically targetable fluorescent indicators. Nat Protoc 2006; 1: 1057-1065.

18. Popko B, Baerwald KD. Oligodendroglial response to the immune cytokine interferon gamma. Neurochem Res 1999; 24: 331-338.

19. Bonora M, Bononi A, De Marchi E, Giorgi C, Lebiedzinska M, Marchi S et al. Role of the C subunit of the FO ATP synthase in mitochondrial permeability transition. Cell Cycle 2013; 12: 674-683.

20. Suski JM, Lebiedzinska M, Bonora M, Pinton P, Duszynski J, Wieckowski MR. Relation between mitochondrial membrane potential and ROS formation. Methods Mol Biol 2012 810: 183-205.

21. Mao C, Kisaalita WS. Determination of resting membrane potential of individual neuroblastoma cells (IMR-32) using a potentiometric dye (TMRM) and confocal microscopy. J Fluoresc 2004; 14: 739-743.

22. Tanaka M, Davidson M, Yen HC, Suenaga S, Tomita K, Nishii T et al. Evidence of ROS generation by mitochondria in cells with impaired electron transport chain and mitochondrial DNA damage. Mitochondrion 2007; 7: 106-118.

23. Higuchi M, Proske RJ, Yeh ET. Inhibition of mitochondrial respiratory chain complex I by TNF results in cytochrome $\mathrm{c}$ release, membrane permeability transition, and apoptosis. Oncogene 1998; 17: 2515-2524.

24. Willems PHGM, Valsecchi F, Distelmaier F, Verkaart S, Visch HJ, Smeitink JA et al. Mitochondrial $\mathrm{Ca} 2+$ homeostasis in human NADH:ubiquinone oxidoreductase deficiency. Cell Calcium 2008; 44: 123-133.

25. Distelmaier F, Visch HJ, Smeitink JA, Mayatepek E, Koopman WJ, Willems PH. The antioxidant Trolox restores mitochondrial membrane potential and $\mathrm{Ca} 2+$-stimulated ATP production in human complex I deficiency. J Mol Med (Berl) 2009; 87: 515-522.
26. Bolster DR, Crozier SJ, Kimball SR, Jefferson LS. AMP-activated protein kinase suppresses protein synthesis in rat skeletal muscle through down-regulated mammalian target of rapamycin (mTOR) signaling. J Biol Chem 2002; 277: 23977-23980.

27. Tyler Wa, Gangoli N, Gokina P, Kim HA, Covey M, Levison SW et al. Activation of the mammalian target of rapamycin (mTOR) is essential for oligodendrocyte differentiation. J Neurosci 2009; 29: 6367-6378.

28. Navikas V, Link H. Review: cytokines and the pathogenesis of multiple sclerosis. J Neurosci Res 1996; 45: 322-333.

29. Huang $\mathrm{Y}$, Erdmann N, Peng H, Zhao Y, Zheng J. The role of TNF related apoptosisinducing ligand in neurodegenerative diseases. Cell Mol Immunol 2005; 2: 113-122.

30. Vladic A, Horvat G, Vukadin S, Sucic Z, Simaga S. Cerebrospinal fluid and serum protein levels of tumour necrosis factor-alpha (TNF-alpha) interleukin-6 (IL-6) and soluble interleukin-6 receptor (slL-6R gp80) in multiple sclerosis patients. Cytokine 2002; 20 86-89.

31. Cammer W, Zhang $H$. Maturation of oligodendrocytes is more sensitive to TNF alpha than is survival of precursors and immature oligodendrocytes. J Neuroimmunol 1999; 97: 37-42.

32. Scolding N. Oligodendrocyte progenitors are present in the normal adult human CNS and in the lesions of multiple sclerosis. Brain 1998; 121: 2221-2228.

33. Wolswijk G. Oligodendrocyte precursor cells in the demyelinated multiple sclerosis spinal cord. Brain 2002; 125: 338-349.

34. Kuhlmann T, Miron V, Cui Q, Wegner C, Antel J, Brück W. Differentiation block of oligodendroglial progenitor cells as a cause for remyelination failure in chronic multiple sclerosis. Brain 2008; 131: 1749-1758.

35. Fünfschilling U, Supplie LM, Mahad D, Boretius S, Saab AS, Edgar J et al. Glycolytic oligodendrocytes maintain myelin and long-term axonal integrity. Nature 2012; 485: 517-521.

36. Ziabreva I, Campbell G, Rist J, Zambonin J, Rorbach J, Wydro MM et al. Injury and differentiation following inhibition of mitochondrial respiratory chain complex IV in rat oligodendrocytes. Glia 2010; 58: 1827-1837.

37. Marchi S, Pinton P. The mitochondrial calcium uniporter complex: molecular components, structure and physiopathological implications. J Physiol 2014; 592: 829-839.

38. Harding AE, Sweeney MG, Miller DH, Mumford CJ, Kellar-Wood H, Menard D et al. Occurrence of a multiple sclerosis-like illness in women who have a Leber's hereditary optic neuropathy mitochondrial DNA mutation. Brain 1992; 115(Pt 4): 979-989.

39. Vanopdenbosch L, Dubois B, D'Hooghe MB, Meire F, Carton H. Mitochondrial mutations of Leber's hereditary optic neuropathy: a risk factor for multiple sclerosis. J Neurol 2000; 247: 535-543.

40. Chen Q, Vazquez EJ, Moghaddas S, Hoppel CL, Lesnefsky EJ. Production of reactive oxygen species by mitochondria: central role of complex III. J Biol Chem 2003; 278: 36027-36031.

41. Szolnoki Z, Kondacs A, Mandi Y, Bodor A, Somogyvari F. A homozygous genetic variant of mitochondrial uncoupling protein 4 exerts protection against the occurrence of multiple sclerosis. Neuromolecular Med 2009; 11: 101-105.

42. Szolnoki Z. Common genetic variants of the mitochondrial trafficking system and mitochondrial uncoupling proteins affect the development of two slowly developing demyelinating disorders, leukoaraiosis and multiple sclerosis. Curr Med Chem 2010; 17: 3583-3590.

43. Szolnoki Z, Kondacs A, Mandi Y, Bodor A, Somogyvari F. A homozygous genetic variant of mitochondrial uncoupling protein 4 affects the occurrence of leukoaraiosis. Acta Neurol Scand 2011; 123: 352-357.

44. Otaegui D, Saenz A, Ruiz-Martinez J, Olaskoaga J. \& López de Munain, A. UCP2 and mitochondrial haplogroups as a multiple sclerosis risk factor. Mult Scler 2007; 13: 454-458.

45. Wu S-B, Wei Y-H. AMPK-mediated increase of glycolysis as an adaptive response to oxidative stress in human cells: implication of the cell survival in mitochondrial diseases. Biochim Biophys Acta 2012; 1822: 233-247.

46. Alers S, Löffler AS, Wesselborg S, Stork B. Role of AMPK-mTOR-Ulk1/2 in the regulation of autophagy: cross talk, shortcuts, and feedbacks. Mol Cell Biol 2012; 32: 2-11.

47. Díaz-Troya S, Pérez-Pérez ME, Florencio FJ, Crespo JL. The role of TOR in autophagy regulation from yeast to plants and mammals. Autophagy 2008; 4: 851-865.

48. Chen Y, Balasubramaniyan V, Peng J, Hurlock EC, Tallquist M, Li J et al. Isolation and culture of rat and mouse oligodendrocyte precursor cells. Nat Protoc 2007; 2: 1044-1051.

49. Bradford MM. A rapid and sensitive method for the quantitation of microgram quantities of protein utilizing the principle of protein-dye binding. Anal Biochem 1976; 72: 248-254. 